\title{
SCALAR CURVATURE OF HYPERSURFACES WITH CONSTANT MEAN CURVATURE IN SPHERES
}

\author{
QIN ZHANG \\ Institute of Mathematics and Software Science, Sichuan Normal University, Chengdu 610066, China \\ e-mail: zhangdiligence@126.com
}

(Received 9 October 2010; revised 13 April 2011; accepted 28 April 2011; first published online 2 August 2011)

\begin{abstract}
Let $M^{n}$ be an $n$-dimensional closed hypersurface with constant mean curvature $H$ satisfying $|H| \leq \varepsilon(n)$ in a unit sphere $S^{n+1}(1), n \leq 8$ and $S$ the square of the length of the second fundamental form of $M$. There exists a constant $\delta(n, H)>0$, which depends only on $n$ and $H$ such that if $S_{0} \leq S \leq S_{0}+\delta(n, H)$, then $S \equiv S_{0}$ and $M$ is isometric to a Clifford hypersurface, where $\varepsilon(n)$ is a sufficiently small constant depending on $n$ and $S_{0}=n+\frac{n^{3}}{2(n-1)} H^{2}+\frac{n(n-2)}{2(n-1)} \sqrt{n^{2} H^{4}+4(n-1) H^{2}}$.
\end{abstract}

2010 Mathematics Subject Classification. Primary 53C42, 53B25.

1. Introduction. Let $M^{n}$ be an $n$-dimensional closed hypersurface with constant mean curvature $H$ in a unit sphere $S^{n+1}(1)$ of dimension $n+1$, denoted by $S$ the squared norm of the second fundamental form of $M^{n}$.

When $H \equiv 0$, Lawson [16], Simons [10] and Chern et al. [8] obtained independently the famous rigidity theorem, which says, if $S \leq n$, then $S \equiv 0$, or $S \equiv n$, i.e. $M^{n}$ is the great sphere $S^{n}(1)$, or the Clifford torus. Further discussions in this direction have been carried out by many other authors $[2,5,7,12,18,19-21]$. In [14], Peng and Terng proved that if the scalar curvature of $M$ is constant, then there exists a positive constant $\alpha(n)$ depending only on $n$ such that if $n \leq S \leq n+\alpha(n)$, then $S \equiv n$. Later, Cheng and Yang [6] improved the pinching constant $\alpha(n)$ to $\frac{n}{3}$. Without the assumption of constant scalar curvature, Peng and Terng [15] proved that if $M^{n}(n \leq 5)$ is a closed minimal hypersurface in $S^{n+1}$, then there exists a positive constant $\alpha(n)$ depending only on $n$ such that if $n \leq S \leq n+\alpha(n)$, then $S \equiv n$. So they proposed the following attractive problem:

Let $M^{n}(n \geq 6)$ be a closed minimal hypersurface in $S^{n+1}$. Does there exist a positive constant $\alpha(n)$ depending only on $n$ such that if $n \leq S \leq n+\alpha(n)$, then $S \equiv n$ and $M$ is isometric to a Clifford torus $S^{k}\left(\sqrt{\frac{k}{n}}\right) \times S^{n-k}\left(\sqrt{\frac{n-k}{n}}\right)$ ?

In [3], Cheng gave a positive answer under the additional condition that $M$ has only two distinct principal curvatures. Later, Hasanis and Vlachos [9] proved that if $M^{n}$ is a compact minimal hypersurface in $S^{n+1}$ with two distinct principal curvatures and the squared norm $S$ of the second fundamental form of $M^{n}$ satisfies $S \geq n$, then $M^{n}$ is a minimal Clifford torus. In [5], Cheng and Ishikawa improved the result of Peng and Terng [15] when $n \leq 5$. Later, Wei and $\mathrm{Xu}$ [17] solved the problem proposed by Peng and Terng [15] for $n=6$ and 7. Recently, we [22] obtained a sharper pinching constant of $S$ for $n \leq 7$ and solved this problem for $n=8$. 
When $M$ is a hypersurface with constant mean curvature, Alencar and do Carmo [1] proved the first rigidity result under the assumption that the traceless second fundamental form is sufficiently bounded. Later, Li [11] extended the result of Peng and Terng [15] for minimal hypersurfaces to the case of hypersurfaces with constant mean curvature. That is, $\mathrm{Li}[\mathbf{1 1}]$ proved the following theorem:

Let $M$ be an n-dimensional closed hypersurface with constant mean curvature $H$ satisfying $|H| \leq \varepsilon(n)$ in a unit sphere $S^{n+1}, n \leq 5$, and $S$ the square of the length of the second fundamental form of $M$. Then there exists a constant $\delta(n, H)>0$, which depends only on $n$ and $H$, such that if $S_{0} \leq S \leq S_{0}+\delta(n, H)$, then $S \equiv S_{0}$ and $M$ is isometric to a Clifford torus $S^{k}\left(\sqrt{\frac{k}{n}}\right) \times S^{n-k}\left(\sqrt{\frac{n-k}{n}}\right)$ if $H=0 ; M$ is isometric to a Clifford hypersurface $C_{1, n-1}=S^{1}\left(\frac{1}{\sqrt{1+\lambda^{2}}}\right) \times S^{n-1}\left(\frac{\lambda}{\sqrt{1+\lambda^{2}}}\right)$ if $H \neq 0$, where $\lambda=\frac{n H+\sqrt{n^{2} H^{2}+4(n-1)}}{2}$ and $\varepsilon(n)$ is a sufficiently small constant depending on $n, S_{0}=$ $n+\frac{n^{3}}{2(n-1)} H^{2}+\frac{n(n-2)}{2(n-1)} \sqrt{n^{2} H^{4}+4(n-1) H^{2}}$.

In [4], Cheng, He and Li proved the above theorem is valid for the case of $n=6,7$. In this paper, we study the case of $n=8$. We prove the following theorem.

THEOREM 1.1. Let $M$ be an n-dimensional closed hypersurface with constant mean curvature $H$ satisfying $|H| \leq \varepsilon(n)$ in a unit sphere $S^{n+1}, n \leq 8$, and $S$ the square of the length of the second fundamental form of $M$. Then there exists a constant $\delta(n, H)>0$, which depends only on $n$ and $H$, such that if $S_{0} \leq S \leq S_{0}+\delta(n, H)$, then $S \equiv S_{0}$ and $M$ is isometric to a Clifford torus $S^{k}\left(\sqrt{\frac{k}{n}}\right) \times S^{n-k}\left(\sqrt{\frac{n-k}{n}}\right)$ if $H=0 ; M$ is isometric to a Clifford hypersurface

$$
C_{1, n-1}=S^{1}\left(\frac{1}{\sqrt{1+\lambda^{2}}}\right) \times S^{n-1}\left(\frac{\lambda}{\sqrt{1+\lambda^{2}}}\right)
$$

if $H \neq 0$, where $\lambda=\frac{n H+\sqrt{n^{2} H^{2}+4(n-1)}}{2}$ and $\varepsilon(n)$ is a sufficiently small constant depending on $n$,

$$
S_{0}=n+\frac{n^{3}}{2(n-1)} H^{2}+\frac{n(n-2)}{2(n-1)} \sqrt{n^{2} H^{4}+4(n-1) H^{2}} .
$$

2. Fundamental formulas. Let $M^{n}$ be an $n$-dimensional hypersurface with constant mean curvature $H$ in an $(n+1)$-dimensional unit sphere $S^{n+1}(1)$. We choose a local orthonormal frame field $e_{1}, \ldots, e_{n+1}$ in $S^{n+1}(1)$, restricted to $M^{n}$, so that $e_{1}$, $\ldots, e_{n}$ are tangent to $M^{n}$. Let $\omega_{1}, \ldots, \omega_{n+1}$ denote the dual coframe field in $S^{n+1}(1)$. Then in $M^{n}, \omega_{n+1}=0$. It follows from Cartan's Lemma that

$$
\omega_{n+1 i}=\sum_{j} h_{i j} \omega_{j} .
$$

The second fundamental form $\alpha$ and the mean curvature $H$ of $M^{n}$ are defined by

$$
\alpha=\sum_{i j} h_{i j} \omega_{i} \omega_{j} e_{n+1}, \quad n H=\sum_{i} h_{i i}
$$


respectively. The connection form $\omega_{i j}$ is characterized by the structure equations

$$
\begin{gathered}
d \omega_{i}+\sum_{j} \omega_{i j} \wedge \omega_{j}=0, \quad \omega_{i j}+\omega_{j i}=0, \\
d \omega_{i j}+\sum_{k} \omega_{i k} \wedge \omega_{k j}=\Omega_{i j}, \\
\Omega_{i j}=\frac{1}{2} \sum_{k, l} R_{i j k l} \omega_{k} \wedge \omega_{l},
\end{gathered}
$$

where $\Omega_{i j}$ (resp. $R_{i j k l}$ ) denotes the curvature form (resp. the components of the curvature tensor) of $M^{n}$. The Gauss equation is given by

$$
R_{\ddot{j} k l}=\left(\delta_{i k} \delta_{j l}-\delta_{i l} \delta_{j k}\right)+\left(h_{i k} h_{j l}-h_{i l} h_{j k}\right) .
$$

Denote by $h_{i j k}, h_{i j k l}, h_{i j k l m}$ components of the first, second and third covariant derivatives of the second fundamental form, respectively. Then

$$
\begin{gathered}
h_{i j k}=h_{i k j}=h_{j i k}, \\
h_{i j k l}-h_{i j l k}=\sum_{m} h_{i m} R_{m j k l}+\sum_{m} h_{m j} R_{m i k l}, \\
h_{i j k l m}-h_{i j k m l}=\sum_{r} h_{i j k} R_{r i l m}+\sum_{r} h_{i r k} R_{r j l m}+\sum_{r} h_{i j r} R_{r k l m} .
\end{gathered}
$$

For any fixed point $p$ in $M^{n}$, we take a local orthonormal frame field $e_{1}, \ldots, e_{n}$ such that

$$
h_{i j}= \begin{cases}\lambda_{i}, & i=j, \\ 0, & i \neq j .\end{cases}
$$

We define the squared norm of the second fundamental form $S$ of $M, f_{3}, f_{4}$ to be

$$
S=\sum_{i, j} h_{i j}^{2}, \quad f_{3}=\sum_{i, j, k} h_{i j} h_{j k} h_{k i}, \quad f_{4}=\sum_{i, j, k, l} h_{i j} h_{j k} h_{k l} h_{l i} .
$$

Then at the point $p$, we have

$$
S=\sum_{i} \lambda_{i}^{2}, \quad f_{3}=\sum_{i} \lambda_{i}^{3}, \quad f_{4}=\sum_{i} \lambda_{i}^{4} .
$$

Since the mean curvature $H$ of $M$ is a constant, using the above equations, we easily get

$$
\begin{aligned}
\frac{1}{2} \triangle S= & \sum_{i, j, k} h_{i j k}^{2}-S(S-n)-n^{2} H^{2}+n H f_{3}, \\
\frac{1}{2} \triangle \sum_{i, j, k} h_{i j k}^{2}= & \sum_{i, j, k, l} h_{i j k l}^{2}+(2 n+3-S) \sum_{i, j, k} h_{i j k}^{2}+3(2 B-A) \\
& +3 n H \sum_{i, j, k} \lambda_{i} h_{i j k}^{2}-\frac{3}{2}|\nabla S|^{2},
\end{aligned}
$$

where $A=\sum_{i, j, k} \lambda_{i}^{2} h_{i j k}^{2}, B=\sum_{i, j, k} \lambda_{i} \lambda_{j} h_{i j k}^{2}$. 
3. Proof of Theorem. At first, we give two lemmas which will play a crucial role in the proof of our theorem. For convenience, we define

$$
\mu_{i j}=h_{i j}-H \delta_{i j}, \quad \mu_{i}=\mu_{i i}, \quad \widetilde{A}=\sum_{i, j, k} \mu_{i}^{2} h_{i j k}^{2}, \quad \widetilde{B}=\sum_{i, j, k} \mu_{i} \mu_{j} h_{i j k}^{2} .
$$

Then

$$
\begin{gathered}
A-2 B=\widetilde{A}-2 \widetilde{B}+2 H \sum_{i, j, k} \lambda_{i} h_{i j k}^{2}+H^{2} \sum_{i, j, k} h_{i j k}^{2}, \\
\sum_{i} \mu_{i}=0, \quad \sum_{i} \mu_{i}^{2}=S-n H^{2} .
\end{gathered}
$$

LEMMA 3.1. Let $M$ be a closed hypersurface with constant mean curvature $H$ in $S^{n+1}(1)$. Then

$$
\begin{aligned}
\sum_{i, j, k, l} h_{i j k l}^{2} \geq & \frac{3}{2}\left\{\left(S f_{4}-f_{3}^{2}-S^{2}+n H f_{3}\right)-\left[S(S-n)+n^{2} H^{2}-n H f_{3}\right]\right\} \\
& +\frac{3\left[S(S-n)+n^{2} H^{2}-n H f_{3}\right]^{2}}{2(n+4)\left(S-n H^{2}\right)} .
\end{aligned}
$$

Proof. From formulae (2.6) and (2.8), we have

$$
\begin{aligned}
h_{i \dddot{j}}-h_{i j i i} & =h_{i j i j}-h_{i j i}=\sum_{m} h_{i m} R_{m i j}+\sum_{m} h_{j m} R_{m i i j} \\
& =\lambda_{i} R_{i j i}+\lambda_{j} R_{j i j}=\left(\lambda_{i}-\lambda_{j}\right) R_{i j i j} \\
& =\left(\lambda_{i}-\lambda_{j}\right)\left(1+\lambda_{i} \lambda_{j}\right) .
\end{aligned}
$$

We define

$$
u_{i j k l}=\frac{1}{4}\left(h_{i j k l}+h_{j k l i}+h_{k l i j}+h_{l i j k}\right) .
$$

Since $h_{i j k l}$ is symmetric in the indices $i, j, k$, from equation (3.3) we obtain

$$
\begin{aligned}
\sum_{i, j, k, l} h_{i j k l}^{2} & =\sum_{i, j, k, l} u_{i j k l}^{2}+\frac{3}{8} \sum_{i, j, k, l}\left(h_{i j k l}-h_{i j l k}\right)^{2} \\
& \geq \sum_{i, j, k, l} u_{i j k l}^{2}+\frac{3}{4} \sum_{i, j}\left(h_{i i j j}-h_{i j i i}\right)^{2} \\
& =\sum_{i, j, k, l} u_{i j k l}^{2}+\frac{3}{2}\left\{\left(S f_{4}-f_{3}^{2}-2 S^{2}+n S-n^{2} H^{2}+2 n H f_{3}\right]\right\} .
\end{aligned}
$$

Since $\sum_{i} h_{i i k l}=0$, we have

$$
\sum_{i, j} \mu_{i} u_{i i j j}=\frac{1}{2}\left(n S-S^{2}-n^{2} H^{2}+n H f_{3}\right)
$$


Since for any $\alpha \in R$,

$$
\sum_{i, j, k, l}\left[u_{i j k l}+\alpha\left(\mu_{i j} \delta_{k l}+\mu_{i k} \delta_{j l}+\mu_{i l} \delta_{j k}+\mu_{j k} \delta_{i l}+\mu_{j l} \delta_{i k}+\mu_{k l} \delta_{i j}\right)\right]^{2} \geq 0,
$$

it follows from equations (3.2) and (3.6) that

$$
\sum_{i, j, k, l} u_{i j k l}^{2} \geq 6 \alpha\left(S^{2}-n S-n H f_{3}+n^{2} H^{2}\right)-6 \alpha^{2}(n+4)\left(S-n^{2} H^{2}\right) .
$$

Letting

$$
\alpha=\frac{S(S-n)+n^{2} H^{2}-n H f_{3}}{2(n+4)\left(S-n^{2} H^{2}\right)},
$$

we have

$$
\sum_{i, j, k, l} u_{i j k l}^{2} \geq \frac{3\left[S(S-n)+n^{2} H^{2}-n H f_{3}\right]^{2}}{2(n+4)\left(S-n H^{2}\right)}
$$

Thus we have finished the proof of Lemma 3.1.

LeMmA 3.2. Let $M$ be an n-dimensional closed hypersurface with constant mean curvature $H$ in $S^{n+1}(1)$, for $n \leq 8$. Then

$$
3(\widetilde{A}-2 \widetilde{B}) \leq 2.34\left(S-n H^{2}\right) \sum_{i, j, k} h_{i j k}^{2} .
$$

Proof. Since $\sum_{i} \mu_{i}=0$ and $\sum_{i} \mu_{i}^{2}=S-n H^{2}=\widetilde{S}$, the following equation can be proved in the same method as in our early paper (Lemma 3.4 in [22]):

$$
\sum_{i(\neq j)}\left(\mu_{j}^{2}-4 \mu_{j} \mu_{i}\right) h_{i j j}^{2}-\mu_{j}^{2} h_{i j j}^{2} \leq 2.34 \widetilde{S}\left(\sum_{i(\neq j)} h_{i j j}^{2}+\frac{1}{3} h_{i j j}^{2}\right), \quad \forall j .
$$

Hence we get

$$
\begin{aligned}
3(\widetilde{A}-2 \widetilde{B})= & \sum_{i \neq j \neq k \neq i}\left[2\left(\mu_{i}^{2}+\mu_{j}^{2}+\mu_{k}^{2}\right)-\left(\mu_{i}+\mu_{j}+\mu_{k}\right)^{2}\right] h_{i j k}^{2} \\
& -3 \sum_{i} \mu_{i}^{2} h_{i i i}^{2}+3 \sum_{i \neq j}\left(\mu_{j}^{2}-4 \mu_{i} \mu_{j}\right) h_{i i j}^{2} \\
\leq & 2 \widetilde{S} \sum_{i \neq j \neq k \neq i} h_{i j k}^{2}+3 \sum_{j}\left\{\sum_{i \neq j}\left[\left(\mu_{j}^{2}-4 \mu_{i} \mu_{j}\right) h_{i j}^{2}-\mu_{j}^{2} h_{i j j}^{2}\right]\right\} \\
\leq & 2.34 \widetilde{S}\left\{\sum_{i \neq j \neq k \neq i} h_{i j k}^{2}+3 \sum_{i \neq j} h_{i i j}^{2}+\sum_{j} h_{i i j}^{2}\right\} \\
= & 2.34 \widetilde{S} \sum_{i, j, k} h_{i j k}^{2} .
\end{aligned}
$$

This completes the proof of Lemma 3.2. 
Proof of Theorem 1.1. Now, we assume

$$
S_{0} \leq S \leq S_{0}+\delta(n, H)
$$

where $S_{0}$ is defined by equation (1.1).

It is not difficult to prove the following elementary inequality (cf. [13]):

$$
\left|\sum_{i}\left(\lambda_{i}-H\right)^{3}\right| \leq \frac{n-2}{\sqrt{n(n-1)}}\left(S-n H^{2}\right)^{\frac{3}{2}} .
$$

Since $S \geq S_{0}$ is equivalent to

$$
\sqrt{n+\frac{n^{3} H^{2}}{4(n-1)}}-\sqrt{S-n H^{2}}+\frac{n(n-2)|H|}{2 \sqrt{n(n-1)}} \leq 0
$$

we have

$$
\begin{aligned}
& S(S-n)+n^{2} H^{2}-n H f_{3} \\
&=-\left(S-n H^{2}\right)\left\{n+n H^{2}-\left(S-n H^{2}\right)\right\}-n H \sum_{i}\left(\lambda_{i}-H\right)^{3} \\
& \geq-\left(S-n H^{2}\right)\left\{n+n H^{2}-\left(S-n H^{2}\right)+\frac{n(n-2)|H|}{\sqrt{n(n-1)}} \sqrt{S-n H^{2}}\right\} \\
& \geq-\left(S-n H^{2}\right)\left\{\sqrt{n+\frac{n^{3} H^{2}}{4(n-1)}}+\sqrt{S-n H^{2}}-\frac{n(n-2)|H|}{2 \sqrt{n(n-1)}}\right\} \\
& \times\left\{\sqrt{\left.n+\frac{n^{3} H^{2}}{4(n-1)}-\sqrt{S-n H^{2}}+\frac{n(n-2)|H|}{2 \sqrt{n(n-1)}}\right\}}\right. \\
& \geq 0 .
\end{aligned}
$$

The following equation can be found in [22] or [11]:

$$
\int_{M}(A-2 B) d M=\int_{M}\left[S f_{4}-f_{3}^{2}-S^{2}+n H f_{3}-\frac{1}{4}|\nabla S|^{2}\right] d M .
$$

Integrating equation (2.13) and $S \times(2.13)$ gives

$$
\begin{gathered}
\int_{M} \sum_{i, j, k} h_{i j k}^{2} d M=\int_{M}\left[S(S-n)+n^{2} H^{2}-n H f_{3}\right] d M . \\
\int_{M} \frac{1}{2}|\nabla S|^{2} d M=\int_{M}\left[S^{2}(S-n)+n^{2} H^{2} S-n H S f_{3}-S \sum_{i, j, k} h_{i j k}^{2}\right] d M .
\end{gathered}
$$

Noticing that

$$
\begin{aligned}
& S(S-n)+n^{2} H^{2}-n H f_{3} \\
& \quad=\left(S-n H^{2}\right)\left(S-S_{0}\right)+n^{2} H^{2}-n H f_{3}-n H^{2} S_{0}+\left(S_{0}+n H^{2}-n\right) S,
\end{aligned}
$$


from equations (3.12) and (3.13), there exists some constant $\alpha_{1}$ such that

$$
\frac{3\left[S(S-n)+n^{2} H^{2}-n H f_{3}\right]^{2}}{2(n+4)\left(S-n H^{2}\right)} \geq\left\{\frac{3\left(S-S_{0}\right)}{2(n+4)}-\alpha_{1} H\right\}\left[S(S-n)+n^{2} H^{2}-n H f_{3}\right]
$$

It follows from equations (3.14), (3.15), (3.18) and Lemma 3.1 that

$$
\begin{aligned}
\int_{M} \sum_{i, j, k, l} h_{i j k l}^{2} d M \geq & \int_{M}\left[\frac{3}{2}(A-2 B)-\frac{3}{2} \sum_{i, j, k} h_{i j k}^{2}+\frac{3}{8}|\nabla S|^{2}\right] d M \\
& +\int_{M}\left[\frac{3\left(S-S_{0}\right)}{2(n+4)}-\alpha_{1} H\right]\left[S(S-n)+n^{2} H^{2}-n H f_{3}\right] d M .
\end{aligned}
$$

From equations (2.14) and (3.2), we have

$$
\begin{aligned}
\int_{M} \sum_{i, j, k, l} h_{i j k l}^{2} d M= & \int_{M}\left[(S-2 n-3) \sum_{i, j, k} h_{i j k}^{2}+\frac{3}{2}|\nabla S|^{2}+\frac{3}{2}(A-2 B)\right] d M \\
& +\int_{M}\left[\frac{3}{2}(\widetilde{A}-2 \widetilde{B})+3(1-n) H \sum_{i, j, k} \lambda_{i} h_{i j k}^{2}+\frac{3}{2} H^{2} \sum_{i, j, k} h_{i j k}^{2}\right] d M .
\end{aligned}
$$

Since $S_{0} \leq S \leq S_{0}+\delta(n, H)$, there exists some constant $\alpha_{2}$ such that

$$
\begin{aligned}
\int_{M} \sum_{i, j, k, l} h_{i j k l}^{2} d M \leq & \int_{M}\left[\left(S-2 n-3+\alpha_{2} H\right) \sum_{i, j, k} h_{i j k}^{2}+\frac{3}{2}(A-2 B)\right] d M \\
& +\int_{M}\left[\frac{3}{2}(\widetilde{A}-2 \widetilde{B})+\frac{3}{2}|\nabla S|^{2}\right] d M .
\end{aligned}
$$

From equations (3.13), (3.15), (3.16), (3.19), (3.20) and Lemma 3.2, we obtain

$$
\begin{aligned}
0 \leq & \int_{M}\left\{\left[S-2 n-\frac{3}{2}+\left(\alpha_{1}+\alpha_{2}\right) H\right] \sum_{i, j, k} h_{i j k}^{2}+\frac{3}{2}(\widetilde{A}-2 \widetilde{B})\right\} d M \\
& +\int_{M}\left\{\frac{9}{8}|\nabla S|^{2}-\frac{3\left(S-S_{0}\right)}{2(n+4)}\left[S(S-n)+n^{2} H^{2}-n H f_{3}\right]\right\} d M \\
\leq & \int_{M}\left[-0.08 S-2 n-\frac{3}{2}+\left(\alpha_{1}+\alpha_{2}-1.17 n H\right) H\right] \sum_{i, j, k} h_{i j k}^{2} d M \\
& +\int_{M}\left[\frac{9}{4} S-\frac{3\left(S-S_{0}\right)}{2(n+4)}\right]\left[S(S-n)+n^{2} H^{2}-n H f_{3}\right] d M \\
\leq & \int_{M}\left[-0.08 S-2 n-\frac{3}{2}+\left(\alpha_{1}+\alpha_{2}-1.17 n H\right) H\right] \sum_{i, j, k} h_{i j k}^{2} d M \\
& +\int_{M}\left\{\frac{9}{4} S_{0}+\frac{9 n+30}{4(n+4)} \delta(n, H)\right\}\left[S(S-n)+n^{2} H^{2}-n H f_{3}\right] d M \\
\leq & \int_{M} G \sum_{i, j, k} h_{i j k}^{2} d M,
\end{aligned}
$$

where $G=2.17 S_{0}+\frac{9 n+30}{4(n+4)} \delta(n, H)-2 n-\frac{3}{2}+\left(\alpha_{1}+\alpha_{2}-1.17 n H\right) H$. 
Since $2.17 n-2 n-\frac{3}{2}<0$ and $|H| \leq \varepsilon(n)$, if $\varepsilon(n)$ is small enough, we can choose $\delta(n, H)$ such that

$$
2.17 S_{0}+\frac{9 n+30}{4(n+4)} \delta(n, H)-2 n-\frac{3}{2}+\left(\alpha_{1}+\alpha_{2}-1.17 n H\right) H<0 .
$$

According to equations (3.21) and (3.22), we infer $\sum_{i, j, k} h_{i j k}^{2} \equiv 0$. Hence, all of the above inequalities are equalities. From equation (3.13) and (3.15), we have $S \equiv S_{0}$ and $M$ is isometric to a Clifford hypersurface. Thus we have finished the proof of Theorem 1.1.

REMARK 3.3. In the proof of Theorem 1.1, the constants $\alpha_{1}$ and $\alpha_{2}$ are chosen so that

$$
\alpha_{1} \geq \frac{3 \delta(n, H)}{3(n+4) H}, \quad \alpha_{2}>\frac{3 S_{0}}{2 n H} \quad \text { if } H>0
$$

and so

$$
\delta(n, H):=\min \left\{\frac{(n+4)(2 n+3 / 2-2.17 n)}{3 n+10}, \frac{2(n+4) H}{3} \alpha_{1}, \frac{2 n H}{3} \alpha_{2}-S_{0}\right\} .
$$

ACKNOWLEDGEMENTS. The author would like to express his gratitude to the referee for his valuable suggestions that have really improved the paper.

\section{REFERENCES}

1. H. Alencar and M. do Carmo, Hypersurfaces with constant mean curvature in spheres, Proc. Amer. Math. Soc. 120(1994), 1223-1229.

2. Q. M. Cheng, The classification of complete hypersurfaces with constant mean curvature of space form of dimension 4, Mem. Fac. Sci. Kyushu Univ. 47 (1993), 79-102.

3. Q. M. Cheng, The rigidity of Clifford torus $S^{1}\left(\sqrt{\frac{1}{n}}\right) \times S^{n-1}\left(\sqrt{\frac{n-1}{n}}\right)$, Comment. Math. Helvetici 71 (1996), 60-69.

4. Q. M. Cheng, Y. He and H. Li, Scalar curvature of hypersurfaces with constant mean curvature in a sphere, Glasgow Math. J. 51 (2009), 413-423.

5. Q. M. Cheng and S. Ishikawa, A characterization of the Clifford torus, Proc. Amer. Math. Soc. 127(3) (1999), 819-828.

6. Q. M. Cheng and H. C. Yang, Chern's conjecture on minimal hypersurfaces, Math. Z. 227(3) (1998), 377-390.

7. S. Y. Cheng and S. T. Yau, Hypersurfaces with constant scalar curvature, Math. Ann. 225(3) (1977), 195-204.

8. S. S. Chern, M. do Carmo and S. Kobayashi, Minimal submanifolds of a sphere with second fundamental form of constant length, in Functional analysis and related fields (Springer, New York, 1970), pp. 59-75.

9. T. Hasanis, T. Vlachos, A pinching theorem for minimal hypersurfaces in a sphere, Arch. Math. 75 (2000), 469-471.

10. H. B. Lawson, Local rigidity theorems for minimal hypersurfaces, Ann. Math. 89 (1969), $179-185$

11. H. Li, Scalar curvature of hypersurfaces with constant mean curvature in spheres, Tsinghua Sci. Technol. 1 (1996), 266-269.

12. A. M. Li and J. M. Li, An intrinsic rigidity theorem for minimal submanifolds in a sphere, Arch. Math. (Basel) 58(6) (1992), 582-594. 
13. M. Okumura, Hypersurfaces and a pinching problem on the second fundamental tensor, Amer. J. Math. 96 (1974), 207-213.

14. C. K. Peng and C. L. Terng, Minimal hypersurfaces of sphere with constant scalar curvature, Ann. Math. Stud. 103 (1983), 179-198.

15. C. K. Peng and C. L. Terng, The scalar curvature of minimal hypersurfaces in spheres, Math. Ann. 266 (1983), 105-113.

16. J. Simons, Minimal varieties in Riemannian manifolds, Ann. Math. 88 (1968), 62-105.

17. S. M. Wei and H. W. Xu, Scalar curvature of minimal hypersurfaces in spheres, Math. Res. Lett. 14 (2007), 423-432.

18. H. W. Xu, A rigidity theorem for submanifolds with parallel mean curvature in a sphere, Arch. Math. (Basel) 61(5) (1993), 489-496.

19. H. W. Xu, On closed minimal submanifolds in pinched Riemannian manifolds, Trans. Amer. Math. Soc. 347(5) (1995), 1743-1751.

20. H. W. Xu, W. Fang and F. Xiang, A generalization of Gauchman's rigidity theorem, Pac. J. Math. 228(1) (2006), 185-199.

21. S. T. Yau, Submanifolds with constant mean curvature, I, II, Amer. J. Math. 96 (1974), 346-366; 96 (1975), 76-100.

22. Qin Zhang, The pinching constant of minimal hypersurfaces in the unit spheres, Proc. Amer. Math. Soc. 138(5) (2010), 1833-1841. 